Whether Cross-Listing, Stock-specific and Market-wide Calendar Events impact Intraday Volatility Dynamics? Evidence from the Indian Stock Market using High-frequency Data

Sobhesh Kumar Agarwalla*

Ajay Pandey

W.P. No. 2012-11-03

November 2012

The main objective of the Working Paper series of IIMA is to help faculty members, research staff, and doctoral students to speedily share their research findings with professional colleagues and to test out their research findings at the pre-publication stage.

\author{
INDIAN INSTITUTE OF MANAGEMENT \\ AHMEDABAD - 380015 \\ INDIA
}




\title{
Whether Cross-Listing, Stock-SPecific And Market-Wide CALENDAR Events impaCt InTRADAY Volatility Dynamics? EVIDENCE FROM THE INDIAN STOCK MARKET USING High-FREQUENCY DATA
}

\author{
Sobhesh Kumar Agarwalla* \\ Ajay Pandey
}

\begin{abstract}
Using high-frequency stock price data, we investigate the effect of various stockspecific and market-wide events on intraday volatility dynamics in the Indian market. Modeling intraday volatility dynamics using FFF regressions, we examine the effect of (a) cross-listing, (b) weekends and holidays, (c) scheduled temporary trading halts, and (d) derivatives' expiry day, on intraday volatility dynamics. We find that Indian stock market exhibits "reverse J" shaped intraday volatility with much higher intraday variation than what has been reported in other markets. The intraday variation is more in the case of large cap stocks relative to small cap stocks. Higher volatility is also observed in the first one-hour of trade after weekends, in the first half-an-hour after the holidays, in the last one-hour of trade before the weekends. Temporary scheduled trading halts cause the volatility to rise when the market re-opens. The stocks, crosslisted elsewhere, exhibit higher volatility in the first 45 minutes of trade relative to other stocks. Volatility of the stocks with derivative contracts increases in the last half-anhour trade on the expiry day, the time period relevant for estimation of the settlement price of the derivative contracts, but not in other time intervals. While our results are mostly along the lines of previous findings, the use of high-frequency data allows us to locate precisely the time-intervals which are affected by the investigated calendar events.
\end{abstract}

Keywords: Intraday volatility, Emerging Market, Cross-listing, Index Maturity

JEL classifications: G12, G14, G15

*Corresponding Author (email: sobhesh@iimahd.ernet.in). Correspondence Address: Wing 7E, Indian Institute of Management Ahmedabad, Vastrapur, Ahmedabad, Gujarat, India 380015. Both the authors are faculty members at the Indian Institute of Management, Ahmedabad. This paper is based on the dissertation of the first author. 


\section{Introduction}

Empirical research across financial markets has noted regularities in intraday behavior of volume and volatility. Typically, both the volatility of returns and volume of trading is found ${ }^{1}$ to be "U-shaped", i.e., more at the beginning and at the close of trading as compared to rest of the trading hours. In some markets, the increase towards the close of trading is less pronounced resulting in so called "reverse J" shaped pattern or even "L shaped" intraday pattern. Researchers have also explored the role of information flow and of the microstructure variables as determinants of intraday volatility.

The market microstructure models such as the ones proposed by Admati and Pfleiderer (1988) and Brock and Kleidon (1992) imply certain intraday dynamics of volume, volatility and spread due to informed traders trading on their information with uninformed traders. In the Admati and Pfleiderer model, the periods of concentrated trading arise endogenously because of the strategic behavior of discretionary liquidity (uninformed) traders and informed traders. According to the model, variability of price changes is higher in periods of concentrated trading, and the intraday trading patterns in financial markets are determined by factors that are exogenous to the model such as the rate of arrival of public information, the amount of nondiscretionary liquidity trading, and the length of time used by discretionary liquidity trader for trading. Brock and Kleidon (1992) also specifically examine the effect of periodic stock market closure on transactions demand and volume of trade, and consequently on bid and ask prices. In their model, the desire of investors to rebalance their portfolios based on perceived (at close) and actual (at open) overnight information results in greater and less elastic transaction demand at open and close than at other times of trading day. However, according to them intraday volatility should not follow any specific pattern as information arrival is constant across the day.

One of the reasons given for high open and close volatility is price variability associated with pricing of overnight information flow (McInish and Wood 1990, Foster and Viswanathan 1990). Market takes some time to incorporate overnight information causing high variance at market opening. Difference in perception and valuation of expected overnight information causes high variance at market close. Empirical studies have attempted to study specific cases of and exceptions to accumulation of information due to trading halts and assimilation of information during trading. Past studies provide mixed evidence on return volatility on Monday and Fridays ${ }^{2}$. We attempt to find whether the high/low volatility on Monday and Fridays can be attributable to the market open (close) surrounding the extended trading halt.

Cross-listed stocks, where the foreign listing is in a market in different time-zone, present a case where the trading continues much after it has stopped for other stocks. Since these

\footnotetext{
${ }^{1}$ Past studies have shown that the intraday return volatility pattern is U-shaped for NYSE, which is a specialist market (Wood et al. 1985), reverse J-shaped for NASDAQ (Chan et al. 1995) and U-shaped for LSE (Abhyankar et al. 1997), which are quote driven markets. Presence of intraday periodicity in return volatility has been documented by many studies in both equity (Wood et al. 1985, Harris 1986), as well as foreign exchange markets (Muller et al. 1990, Andersen and Bollerslev 1998a).

2 Past studies relating to DM-US\$ return volatility (Andersen and Bollerslev 1998a) have found the Monday morning effect to be insignificant and Friday afternoon to be borderline significant with a negative coefficient. Using daily data, Kiymaz and Berument (2003) had documented highest return volatility on Mondays for Germany and Japan, on Fridays for Canada and the US and on Thursday for the UK market.
} 
stocks are traded overnight (in foreign market), relatively more recent price quotes are available and hence variance of price at opening should be low for these stocks, assuming that information can flow freely (Amihud and Mendelson 1991). Amihud and Mendelson (1987) observe that pricing errors at open are lower for cross-listed stocks vis-á-vis other stocks and conclude that available sequence of transaction prices from the trading day in other markets facilitates faster price discovery for cross-listed stocks.

Another phenomenon of empirical interest in the context of intraday dynamics is the effect of expiry of derivative contracts on prices, volume and volatility. Stoll and Whaley $(1986 ; 1987$; 1991) find significant increase in trading volumes in index stocks during last hour on expiry day of S \& P 500 future contracts. More recently, Chow et al. (2003) find abnormal effect on price and volatility (but not on volume) of the underlying stocks on expiration day in the Hong Kong market. Alkeback and Hagelin (2004) find high volumes but no price distortions in Swedish market. Vipul (2005), based on low-frequency data from Indian stock market, notes that the price and volatility are sometimes distorted near expiration day in the Indian market due to unwinding of cash positions by arbitrageurs in cash markets.

To the extent a specific institutional context can reduce or increase information asymmetry between informed and uninformed traders or can reduce or enhance the incentives for trading by informed and uninformed traders; it can potentially impact the intraday dynamics of trading and hence volatility, if information asymmetry is indeed the primary driver of observed intraday volatility dynamics. In the context of Indian stock markets, this study investigates the effect of cross-listing of stocks, short and extended trading halts, and expiry of derivative contracts on intraday volatility dynamics using high-frequency data. While there are numerous studies on the impact of cross-listing, trading vs. non-trading hours, scheduled trading breaks, and expiry day on price discovery process across different asset markets using low-frequency data, there are very few empirical works on the effect of these events based on high-frequency data. Use of high-frequency data has the advantage of being more precisely able to locate in time the impact of any event on intraday dynamics.

In order to examine the effect of the above stock-specific and market-wide calendar events on the intraday volatility dynamics, we model the intraday volatility dynamics of Indian stock markets using Flexible Fourier Form (FFF) regression model ${ }^{3}$ proposed by Andersen and Bollerslev (1998a). As mentioned earlier, it has been found that the return volatility varies systematically over the trading day across different asset markets. Andersen and Bollerslev (1998a) argued for integrating the effect of public announcements, of trading (market microstructure) induced intraday periodicities, and of volatility persistence (ARCH effect) while modeling variance of asset returns at higher frequencies. Previous research has documented the intraday dynamics of volume and volatility in the Indian stock market (Agarwalla 2010) and this paper can be seen as a natural extension of that stream of research.

Remainder of this paper is organized as follows. Section 2 gives an overview of the institutional details of National Stock Exchange of India (NSE) and describes our data

\footnotetext{
${ }^{3}$ Another method that has been used in earlier studies (for instance, Evans and Speight 2010) is the cubic spline approach advocated by Taylor (2004a;b) wherein the intraday periodicity is captured by fitting three degree polynomials between predefined points (called 'Knots'). Cubic spline methodology does not impose a smooth pattern of intraday volatility and allows for sharp peak and troughs (Evans and Speight 2010) provided such peaks and troughs exist.
} 
set. Section 3 discusses the observed intraday patterns and periodicity in return volatility in Indian market and describes the methodology used to model the intraday volatility dynamics. Results are discussed in section 4 and section 5 concludes.

\section{Institutional Details of the National Stock Exchange of India (NSE) and the Sample}

The Indian capital market ranked tenth in the world by total traded value and seventh in the world by market capitalization in 2010 (ISMR 2011). NSE (National Stock Exchange of India), globally ranked 4th by number of trades in equity shares and 2nd in terms of number of contracts traded in single stock futures in 2009, had a domestic market share of $75 \%$ in the cash market segment and $100 \%$ in the individual futures and options segment in 2010-11 (ISMR 2010). Around 1400 companies were listed on the NSE during 2009-10 out of which 1301 companies traded for more than 100 days.

The average trading volume of the cash market segment of NSE had increased from $\$ 192.3$ billion (₹ 8,390 billion) in $1999-2000$ to $\$ 916.7$ billion (₹ 41,380 billion) in $2009-10$. The average daily trading volume was ₹ 169.6 billion (US $\$ 3,757$ million) during 2009-10. However, significant volume at the NSE was contributed by day traders who usually square off their position before the close of the day ${ }^{4}$.

NSE offers a fully automated screen-based order-driven trading system, and had no opening ${ }^{5}$ or closing call auction during the period covered in this study. The normal market timings were 9:55 hours to 15:30 hours and was unchanged ${ }^{6}$ during the entire period covered in this study.

NSE employs an Open Electronic Continuous Auction Limit Order Book (OECLOB) mechanism known as National Exchange for Automated Trading (NEAT) that relies completely on unfilled limit orders for liquidity. NSE uses satellite communication to enable trading across the country. The system matches the orders on a "price-time" priority and the uniform response time per trade is less than 1.5 seconds (NSE 2008). The market is highly transparent as NSE provides detailed online real-time trading information.

During the period covered in the study, the derivatives market included both single stock (only American) futures and options ( F \& O) contracts and F \& O contracts (only European) on indices. The settlement price for the contracts on individual stocks was based on the closing price of the underlying assets. Similarly, for contracts on indices, the final settlement price was determined using the closing price of the constituent stocks on the expiry days of the derivative contracts. The closing price of a stock for the above purposes was calculated using the weighted average price of all trades executed during last 30 minutes of trading on the expiry days.

\footnotetext{
${ }^{4}$ The percentage of trading contributed by day traders has increased over the period. The average volume contributed by the day traders for our sample stocks was $28.1 \%$ in 2002 and $42.2 \%$ in 2009 .

${ }^{5}$ Call auctions were later introduced on 18 October 2010.

${ }^{6}$ The market timings were later changed from beginning of 2010.
} 


\section{Data}

The study covers high frequency trading data of 500 most liquid stocks listed at NSE over 9 years (2001-2009). Stocks were selected based on liquidity criterion, measured as the total number of days during which the stocks had traded during the period. In order to consider continuity $^{7}$, only those stocks were considered which were listed before 1 January 2001 and continued to be listed till 31 December 2009. The period covered is from January 2001 to December 2009, and includes 2230 trading days ${ }^{8}$. We restricted our analysis to December 2009 because there was a major microstructure change in the Indian market from January 2010 when the trade timings were extended (advanced) by 55 minutes. Inclusion of period after January 2010 would have made direct comparison of intraday dynamics with the previous period difficult besides having potential confounding effect on intraday dynamics.

The market capitalization for all the stocks included in the study had increased from ₹ $5,647.54$ billion in 2001 to ₹ 17,277 billion in 2009 . The average daily turnover for the entire sample had increased from ₹ 21.3 billion in 2001 to ₹ 94.96 billion in 2009. The average number of transactions per trading day for the sample had increased from 0.50 million in 1999 to 3.56 million trades in 2007. In terms of trade volume, the average number of shares traded per day had increased from 80.9 million in 1999 to 435.1 million in 2007.

Trade data, index constituents and changes therein, holidays, and other market related information were obtained from the NSE. Other company specific data were obtained from CMIE Prowess.

Out of 500 stocks in our data set, 96 stocks were cross-listed at some point of time during the period. Most of these stocks were listed on LSE, NYSE or on Luxembourg Stock Exchange.

There were no lunch breaks at the NSE. However, NSE used to have a short trading halt twice a year (from 2001 to 2008) for around 10 trading days a year (total 167 trading days), due to disruption in satellite services (referred to as sun-outage breaks). To make up for the lost time, the normal market timings were extended for an equal duration. We have ignored the duration of trading halt and considered the extended trade timings for the purpose of allocating the returns of various five minutes to the 67 intervals. Our model estimates the change in volatility (if any), before and after the trading halt. There were 447 trading-Mondays and 441 trading-Fridays. Number of trading days immediately preceding a holiday(s) and immediately following holiday(s) were 86 and 78 respectively $^{9}$.

NSE had futures and options contracts on six indices during the period of the study. The indices, their respective trading commencement dates and the number of constituent expiry-days in the sample were S \& P CNX Nifty (June 2000, 4466 days), CNX Nifty Junior $^{10}$ (June 2007, 882 days), CNX 100 (June 2007, 1850 trading days), Nifty Midcap 50

\footnotetext{
${ }^{7}$ We thereby ignored all stocks which got listed (after IPO) during the period, since it has been noticed that newly listed stocks are marked by high volume and volatility during the initial trading days.

${ }^{8}$ We exclude days when the market was open for shorter intervals, especially on weekends (special trading sessions, mainly for verifying upgrades in trading systems), holidays (e.g. Diwali), or were open during unusual time (budget days). Further, some days for which the trade files supplied by NSE were incomplete (corrupted) were also excluded.

${ }^{9}$ The difference is because of holidays falling on Friday (or Monday) and hence followed (or preceded) by a holiday.

${ }^{10}$ Derivatives contracts on CNX Nifty Junior and CNX 100 indices stopped trading from July 2009.
} 
(October 2007, 961 days), Bank Nifty (June 2005, 486 days) and CNX IT (September 2003, 926 days). 153 stocks in our sample, with 8132 expiry days, were underlying for individual stocks' derivatives market.

Our model uses returns (and volatility) of five-minute intervals. The 335 minutes of trading time at NSE, starting from 9:55 hours to 15:30 hours ${ }^{11}$, was divided into 67 fiveminute intervals. To calculate the five-minute returns, we first computed log returns of each minute using the last trading price of each minute. The five minute returns were obtained by summing the returns of each minute during the interval. Return of the first minute after a trading break of more than 15 minutes were ignored since the return of interval would then have capture information flow during a longer non-trading period causing higher volatility. Returns of the first minute of the day were computed using market opening prices, thereby ignoring overnight returns.

Intraday periodicities of the volatility may be different across stocks based on their liquidity characteristics. Therefore, considering market capitalization as a proxy for liquidity, we have repeated our analysis by classifying the stocks into five quintiles based on their market capitalization at the end of each year (referred to as Mcap-1 to Mcap-5).

\section{Modeling Intraday Volatility - Fourier Flexible Form Re- gression (FFF)}

\subsection{Intraday Patterns and Periodicity in Indian Market}

Figure 1 shows the intraday patterns of standardized trade size, number of trades, volume and the absolute return (volatility) in Indian market. Whereas the volatility is "Reverse-J" shaped, the trade size, the volume and the number of trades are U-shaped through the trading hours in Indian market. The volatility is higher both at the open and close as compared to other intervals. However, the volatility at the close is flatter relative to other markets. For the Nikkei-225 absolute returns series, the absolute returns at the close are higher than at the open (Andersen et al. 2000).

The magnitude of intraday variation is higher in the Indian capital markets. Whereas, in other markets (Nikkei-225 and S \& P 500) the volatility at intraday peak is around twice the volatility at intraday low, in India it is almost four times. The intraday five-minute absolute returns range from $0.05 \%$ to $0.11 \%$ in the case of Nikkei-225 (Andersen et al. 2000) and from $0.05 \%$ to $0.10 \%$ in the case of S \& P 500 (Andersen and Bollerslev 1997a), but, as can be seen in Figure 1, the average intraday volatility of the 500 stocks in our sample range from $0.3 \%$ to $1.2 \%$. The peak five-minute volatility levels in India are significantly higher than in other markets.

Figure 2 (the red colored lines) shows the correlograms for average five-minute absolute (demeaned) returns for the entire sample and for each M-cap quintiles. The serial auto-

\footnotetext{
${ }^{11}$ After the normal closing at 15:30 hours NSE reopens for a closing session of 10 minutes from 15:50 to 16:00 hours to provide an opportunity to investors to close their trading positions. Only market orders are allowed in the session and the settlement is done at a single price computed from the pending price quotes, as in case of an auction market. We exclude such trades since there is no volatility observed in price during this period.
} 
correlation is measured up to 603 lags (covering 9 trading days) for the series consisting of 149,410 (2230 trading days * 67 intervals) 5-minute intraday averages of returns volatility of the stocks in the sample (quintile). It can be seen that all the correlograms display a series of U-shapes, with each U-shape covering 67 lags (one trading days) implying systematic cycle of auto-correlations ${ }^{12}$. The U-shaped patterns are in line with the evidence in US equity and foreign exchange markets documented by Andersen and Bollerslev (1997b; 1998b) and Andersen et al. (2000). The level of autocorrelations and the depth of the Ushape patterns in the correlograms decreases with reduction in the market capitalization. However, the level of autocorrelations is very high as compared to other asset markets. It should be noted that the previous studies have calculated autocorrelation of the volatility of a particular return series (index/currency returns, etc.) but we have calculated autocorrelations using the series of average volatility across various stocks during a particular interval.

\subsection{Modeling Intraday Volatility}

Strong intraday periodicity in the volatility, as exhibited in the Indian market, has also been documented by various studies on foreign exchange and equity returns. For reasons mentioned earlier, we have used the Flexible Fourier Form (FFF) Regression model that was introduced by Gallant (1981) and has been widely used by Andersen and Bollerslev (1997a;b; 1998a;b) for similar studies. The model incorporates the intraday volatility component as well as a daily volatility component and adapts well to the smooth cyclical pattern of intraday volatility. The FFF regression model assumes that intraday patterns are largely stable over time and are independent of the serial correlation in daily volatility. It is of the following form:

$$
R_{m, t, n}=\sigma_{m, t} . S_{t, n} . Z_{m, t, n}
$$

$R_{m, t, n}$ is the $\log$ return of stock $m$ at the $n^{\text {th }}$ five-minute interval of day $t . \sigma_{m, t}$ is the daily volatility factor for stock $m$ on day $t . S_{t, n}$ represent components associated with intraday volatility and any other predictable factor affecting the expected return volatility over the $n^{t h}$ interval; and $Z_{m, t, n}$ is the i.i.d. unit variance, zero mean term.

Log transformation of five-minute absolute returns is used for analysis as it removes any outliers and yields more robust results. Squaring, taking log and rearranging equation (1) results in the following expression:

$$
2 \log \left|R_{m, t, n}\right|-\log \sigma_{m, t}^{2}=c+2 \log S_{t, n}+u_{m, t, n}
$$

where $c=E\left[\log Z_{m, t, n}^{2}\right]$ and $u_{m, t, n}=\log Z_{m, t, n}^{2}-E\left[\log Z_{m, t, n}^{2}\right]$

Equation (2) presents the deviation between the intraday squared returns and daily volatility component to be solely dependent upon the intraday volatility component. In

\footnotetext{
${ }^{12}$ Presence of intraday periodicity in return volatility has been documented by many studies in both equity as well as foreign exchange markets. Wood et al. (1985), and Harris (1986) document intraday periodicity for equity markets and Muller et al. (1990) and Andersen and Bollerslev (1998a) for foreign exchange markets.
} 
order to make the regression represented by equation (2) operational, observable parameterization of the daily volatility component $\sigma_{m, t}$ and of the intraday component $S_{t, n}$ is needed.

In the model $\sigma_{m, t}$ the a-priori estimate of the daily volatility, controls for the source of systematic volatility movements. Following Andersen and Bollerslev (1997a;b; 1998a), we estimate the daily volatility $\sigma_{m, t}$ with a $\operatorname{GARCH}(1,1)$ model using returns during trading hours (335 minutes a day) rather than close to close returns since the objective is to estimate volatility during trading hours. We then normalize the daily volatility by a factor $\sqrt{N}$ to generate proportionate intraday measure to be used in the regression, where $N$ is the number of intraday returns, 67 in our case. This is unlike Andersen and Bollerslev (1998a), wherein a constant estimate $\hat{\sigma}$ for $\hat{\sigma}_{m, t}$ was used, which eliminates the generated regressor problem. Andersen and Bollerslev study models volatility of a single time-series whereas in this study, our objective is to model volatility across stocks.

We also replace the five-minute absolute return, $\left|R_{m, t, n}\right|$ with demeaned absolute returns $\left|R_{m, t, n}-\bar{R}_{m}\right|$, where $\bar{R}_{m}$ is the mean five-minute returns of each company over the entire period $^{13}$. This adjustment helps control the long-term trend in the returns series of each stock. Since the standard deviation dominates the mean of returns particularly for hifrequency time-series, this assumption is relatively innocuous and is not very sensitive to minor misspecification of the conditional mean (Andersen and Bollerslev 1998a).

As regard the intraday volatility component $S_{t, n}$, we impose a parametric representation of the form $f(\theta ; t, n)$, in the resulting equation (3) below. The regressand in equation (2) can be represented as $X_{m, t, n}$ defined as -

$$
X_{m, t, n} \equiv 2 \log \left|R_{m, t, n}-\bar{R}_{m}\right|-\log \hat{\sigma}_{m, t}^{2}+\log N=c+f(\theta ; t, n)+\hat{u}_{m, t, n}
$$

Here $\hat{u}_{m, t, n}$ is a stationary error term. The explanatory variables on the right hand side are determined by FFF in the following form:

$$
f(\theta ; t, n)=\mu+\sum_{k=1}^{K} \lambda_{k} I_{k(m, t, n)}+\sum_{p=1}^{P}\left[\delta_{c, p \cdot \cos }\left(\frac{2 \pi p n}{N}\right)+\delta_{s, p} \cdot \sin \left(\frac{2 \pi p n}{N}\right)\right]
$$

Where, $\theta$ is the vector of right hand side coefficients. Sinusoid terms capture the smooth variations in intraday pattern. The $I_{k(m, t, n)}$ terms represent $K$ dummy variables each representing specific events (described in subsection 3.3) occurring in the $(t, n)$ interval for stock $m$. Parameter coefficients $\lambda_{k}$ quantify the impact of a specific event, whereas the overall shape of intraday pattern is captured by $\delta$ coefficients. Parameters $\mu, \lambda_{k}, \delta_{c, p}$ and $\delta_{s, p}$ are fixed coefficients. Stationary error term $\hat{u}_{m, t, n}$ yields consistent estimates for $\theta$, if the average intraday fluctuations are correctly specified which could be ensured by expanding the order of $P$. The number of sinusoid terms is decided by increasing the number of terms until the coefficients become insignificant. The dummy variables included in the model, along with the interpretation of the results are discussed next.

\footnotetext{
${ }^{13}$ This makes our equation (1) as follows: $R_{m, t, n}=\bar{R}_{m}+\sigma_{m, t} . S_{t, n} . Z_{m, t, n}$
} 


\subsection{Specific Events of Interest}

In earlier empirical studies, dummy variables have been generally considered to capture specific events such as macro-economic announcements and various calendar effects. However, Engle and Sokalska (2012) raise serious objections towards using the FFF framework to capture the effect of macroeconomic news announcements on the intraday volatility. According to them, most important macroeconomic announcements happen before the stock market opens and in other cases, the exact timings of the majority of them is difficult to forecast. They also challenge the assumption that the strength and pattern of market's response to news would be same for similar events across time. Although our model is still subject to the second limitation, but it is relatively free of the first limitation as the events and microstructure effects being investigated by us are related to specific calendar time and our interest is not in informational events. We have used dummy variables to find the effects of following calendar events related to specific time intervals ${ }^{14}$. Each dummy variable covers a period of 15 minutes (3 five-minute intervals), and most of the events are studied using four dummies (to cover a period of one hour). The events examined for their effect on intraday volatility dynamics are:

i. Effect of cross-listing of Stocks on opening and closing volatility: Eight dummy variables $\left(4^{*} 2\right)$ are created to capture the effect of cross-listing during the first and last hour of trading.

ii. Weekends and holidays effect ${ }^{15}$ : Eight dummy variables are included to represent first trading hour on Monday (market open) and last trading hour on Friday (market close). Similarly, eight dummy variables are included to represent the market open following holidays and market close preceding the holidays.

iii. Sun-Outage halt: We include two dummy variables, each representing a 15minutes period immediately before and after the halt to measure the change in volatility surrounding the short trading halts.

iv. Derivatives' expiry day effect: In order to identify the impact of any concerted effort to influence the settlement price of derivative contracts (with individual stocks or indices as underlying) on the expiry day, we include four dummy variables that represents the last one hour of trading on expiry days of derivatives contracts.

v. Opening and closing volatility of stocks acting as underlying for derivatives: The single stock futures contract market in India was very liquid. We expect a faster price discovery of actual (perceived) overnight information at the open (and the close) for

\footnotetext{
${ }^{14}$ We name the four dummy variables (to cover the first trading hour) used to measure the market opening phenomena as 'O1-O4'. Each of these dummies covers three five-minute intervals (15 minutes). 'O1' refers to time interval 1-3 (corresponding to the actual time interval 9:55 to 10:10 hours), 'O2' refers to time interval 4-6 (10:10 to 10:25 hours), and so on. Similarly, each of the four dummy variables (that cover the last trading hour) used to estimate the market closing phenomena (named ' $\mathrm{C} 1$ ' to ' $\mathrm{C} 4$ '). ' $\mathrm{C} 1$ ' refers to the last three time intervals i.e. 65-67 (corresponding to actual time 15:15 to 15:30 hours), 'C2' refers to interval 62-64 (15:00 to 15:15 hours), and so on. By taking four dummy variables (instead of one), we are able to analyze the effect of various phenomena at a micro level.

${ }^{15}$ We have not considered generalized days of the week effect, because past studies have found such effects to be insignificant (Andersen and Bollerslev (1998a) for DM-US\$ returns). Further, the day-of-the-week effect, unless associated with specific announcements associated with specific week-day, is void of any economic justification.
} 
stocks acting as underlying for individual stock derivative markets because of the parallel price discovery process in the derivative market. It is therefore interesting to observe the volatility dynamics of such stocks at market open and close vis-á-vis other stocks. Eight dummy variables are included to represent the first and the last hour of trading of these securities.

\section{Empirical Results}

Regression represented by equation (3) is estimated by simple Ordinary Least Square (OLS) method. The plots in Figure 3 show the average of the actual absolute demeaned fiveminute returns and corresponding estimates obtained from the FFF regression. The six plots represent the curve for the entire sample and for each of the five quintiles. The figures indicate that the FFF regression and the associated choice of parameters in our model provide a good approximation to the average intraday pattern ${ }^{16}$. To obtain the predicted (absolute) return series from the regression equation, we apply the following equation:

$$
\left|R_{m, t, n}-\bar{R}_{m}\right|=N^{-\frac{1}{2}} \cdot \hat{\sigma}_{m, t} \cdot \exp \left(\frac{f(\theta ; t, n)}{2}\right) \cdot \exp \left(\frac{\hat{\mu}_{m, t, n}}{2}\right)
$$

In spite of the seemingly satisfactory fit from the regression approach above, the usefulness of the FFF procedure as a method for filtering out the intraday market microstructure effects ultimately depends upon the ability of the approach to account for the repetitive pattern of the auto-correlations (Andersen et al. 2000). The six plots in figure 3 draw the correlograms for actual and filtered absolute five-minute demeaned returns i.e. $\left|R_{m, t, n}-\bar{R}_{m}\right|$ and $\left|R_{m, t, n}-\bar{R}_{m}\right| / S_{t, n}$ together, averaged across various stocks in the entire sample and in each M-cap quintiles. The filtered series has a smooth decay indicating that the FFF model is able to capture the periodicity in the absolute returns ${ }^{17}$.

Coefficients of the regression parameters are shown in Table 1. We estimate equation (3) separately for M-cap quintiles. The point estimates associated with regular dummy variables in equation (4) can be readily interpreted. A coefficient of unity tantamounts to increase by a multiplicative factor of $e^{\frac{1}{2}} \approx 1.65$ in volatility, i.e. instantaneous increases in the volatility by $65 \%$ of typical volatility associated with the corresponding interval (relative and not absolute). In the remaining part of this section, we discuss the results on each of the specific events where we expected some effect on intraday dynamics of stock volatility based on theoretical arguments or observed empirical regularities elsewhere.

Cross-listing of Stocks: We find, as reported in Table 1, that the volatility is significantly higher during the initial 45 minutes of trading for cross-listed (vis-á-vis other stocks) large M-cap stocks (Mcap-4 and Mcap-5), whereas it is lower for the smaller cap cross-listed stocks. Mcap-5 group of stocks, which has the maximum number of cross-listed stock (more than the numbers of all other quintile groups put together), experiences $18 \%, 22 \%$ and $13 \%$ more volatility during the first three 15-minutes intervals respectively. We expected the ini-

\footnotetext{
${ }^{16}$ Since the approach provides a non-parametric fit to the overall intraday pattern, individual point estimates are generally void of direct economic interpretation. An excellent overall fit is evident from the figure, which shows the success of the model in estimating intraday periodicity with remarkable exactness.

17 The small spikes after every $67^{\text {th }}$ lag could be removed by increasing the number of sinusoidal terms.
} 
tial volatility to be lower for cross-listed stocks since the price discovery process continues for them in other markets when the Indian markets were closed. However, our results are contrary to this expectation. Cross-listed stocks belonging to lower market cap quintiles exhibit less volatility at the opening. One possible reason of our findings may be due to the possibility that prices of these stocks are primarily discovered in the domestic market and not in foreign markets.

Weekends and Holidays: We find, as reported in Table 1, that the volatility is significantly higher at market opening following Monday (all the four 15-minute intervals) and Holidays (first two 15-minute intervals) vis-á-vis other trading days. Volatility during the first 15-minutes of trading on Monday and post the holidays are $10.2 \%$ and $9.8 \%$ higher than other days of the week respectively. The increase in the volatility at market open after weekends (holidays) reduces over the four (two) 15-minutes intervals. The effect is similar across all the quintiles, except for the first quintile (smallest market cap in our sample, Mcap-1). The volatility is also significantly higher during the last trading hour before weekends (4\%). However, there is no difference in the volatility during the last trading hour before holidays vis-á-vis other days. We find the opening effect to be more pronounced in the case of large size firms and the closing effect to be more pronounced in the case of small market-cap stocks. Past studies provide mixed evidence on return volatility on Monday and Fridays. Our results for Indian market attempts to answer whether the change in volatility on Mondays and Fridays can be attributable to specific intraday time interval.

Temporary trading halt (Sun-outage): The regression coefficient for the period before the temporary halt are insignificant (marginally negative), but the one associated with the reopening of market is positive and significant (instantaneous increase of $9 \%$ for the entire sample). The coefficients are positive and significant for mcap2- mcap5 groups, and are higher for large capitalization stocks (16\%, 9.3\% for Mcap-5 and Mcap-4 respectively). The increase in volatility after the trading halt are in line with the market microstructure theory related to price discovery process of new information arrived during the trading halt in markets where there is an asymmetry in the information level of various traders. Our finding is also partially consistent with the intraday volatility pattern at the Tokyo stock exchange where the intraday volatility exhibits a doubly U-shaped pattern and the two U-shapes were associated with the two trading sessions on either side of the lunch break (Andersen et al. 2000).

Derivative Expiry: We notice a significant increase in volatility during the market close of $\mathrm{F} \& \mathrm{O}$ expiry days for stocks acting as underlying for individual derivatives contract vis-á-vis other stocks. The volatility is around $34.2 \%$ higher during the last 15 minutes (C1) i.e. 15:15 to 15:30 hours and around 57.7\% higher during the preceding 15 minutes (C2) i.e. between 15:00 hours to 15:15 hours. The effect during C3 is significant only for Mcap-5 quintile (effect is $6.6 \%$ ) and insignificant for $\mathrm{C} 4$ across all groups. It is quite interesting that the much of the increase in volatility is only during the last 30 minutes, the prices of period relevant for determining the settlement price. The significant high volatility at the expiry days in the Indian market has been documented earlier (Vipul 2005), but our findings bring out the fact that the high volatility is mainly during the last 30 minutes of trading.

The expiry day effect is comparatively higher for stocks in the stocks belonging to the third (42.9\% and $76.4 \%$ for $\mathrm{C} 1$ and $\mathrm{C} 2$ respectively) and fourth M-cap (37.7\% and $53.3 \%$ ) 
quintiles. One possible reason for lower estimates of stocks belong to Mcap-5 group is that relatively larger number of stocks belonging to Mcap-5 group forms part of various indices which are modeled together. Some part of the increase in volatility on these stocks may have been captured by dummy variables representing constituents of various indices. Further the indices are not mutually exclusive and most of the large cap stocks form part of more than one index ${ }^{18}$.

Most of the dummy variables representing expiry days of Nifty, Bank-Nifty, CNX-100, CNX-IT and Nifty-Midcap (all indices except Nifty-Junior) constituent stocks have positive (and significant) coefficients further adding to the magnitude of increase in the volatility during the last 30 minutes of trading on expiry days.

\section{Conclusion}

Both intraday volatility pattern and the overall daily volatility level are required for realtime decision-making and estimating the effects of certain time-specific phenomena as intraday periodicities in volatility have been well documented across different financial markets. FFF framework brings together the three separate components viz. daily volatility factors, intraday time of the day effect, and effects of various time specific phenomena to assess the joint and marginal predictive power of each component. In the context of the Indian stock market, we model intraday volatility dynamics and investigate the effect of cross-listing of stocks, scheduled short trading halts, holidays and weekends, and derivative expiry on the intraday volatility dynamics.

We find that cross-listed stocks exhibit higher volatility during the first 45 minutes of trading at open, which probably indicates that the price discovery of these stocks takes place predominantly in the domestic (Indian) market. As far as scheduled trading breaks due to weekends and holidays are concerned, we find that there is an increase in return volatility during the first hour (30 minutes) of trading at market opening following weekends (holidays). The increase in the volatility gradually reduces over the first hour of trading. The volatility is relatively higher during the last hour of trading before weekends, but not before holidays. Higher volatility at market open and gradual reduction over the opening hour may be attributable to the price discovery process of information releases over the weekends. During trading halts due to sun-outage, we find that the market experiences an increased volatility when it re-opens after the trading halt, but not before the trading halt. This pattern is partially similar to that observed by Andersen et al. (2000) for the Tokyo Stock Exchange.

Finally, we find that the stocks on which derivatives contracts are listed or those which form part of various indices, experience higher volatility during the last 30 minutes of trading on contract expiry days indicating that substantial price movements during the period may be induced by attempts to influence settlement prices. Our results suggests implementation of measures to reduce volatility at market close, (for example, use of call auction sessions) and increased surveillance may be required to reduce the scope of any activities that can

\footnotetext{
${ }^{18}$ For example, out of the 11 stocks in our sample that were constituent of Bank Nifty at some point of time, five stocks were also part of the S \& P CNX Nifty, and out of 13 stocks that were part of CNX-IT Index, five stocks were part of S \& P CNX Nifty index.
} 
result in rigging of settlement prices of future and option contracts. Our results on the effect of derivative expiry of volatility are consistent with findings of Stoll and Whaley (1987) and could be a matter of concern for the exchanges and the regulators.

Most of our results do not vary significantly when we split the entire sample into five quintiles on the basis of market capitalization, a surrogate for the liquidity characteristics of stocks. 


\section{References}

Abhyankar, A., Ghosh, D., Levin, E., and Limmack, R. (1997). Bid-ask spreads, trading volume and volatility: Intra-day evidence from the london stock exchange. Journal of Business, Finance and Accounting, 24(3):343-362.

Admati, A. and Pfleiderer, P. (1988). A theory of intraday patterns: volume and price variability. Review of Financial Studies, 1(1):3-40.

Agarwalla, S. K. (2010). Intraday activity patterns and market microstructure effects in Indian capital markets: An empirical investigation using high frequency data. $\mathrm{PhD}$ thesis, Indian Institute of Management Ahmedabad.

Alkeback, P. and Hagelin, N. (2004). Expiration day effects of index futures and options: evidence from a market with a long settlement period. Applied Financial Economics, 14(6):385-396.

Amihud, Y. and Mendelson, H. (1987). Trading mechanisms and stock returns: An empirical investigation. The Journal of Finance, 42(3):533-553.

Amihud, Y. and Mendelson, H. (1991). Volatility, efficiency, and trading: Evidence from the japanese stock market. The Journal of Finance, 46(5):1765-1789.

Andersen, T. G. and Bollerslev, T. (1997a). Heterogeneous information arrivals and return volatility dynamics: Uncovering the long-run in high frequency returns. The Journal of Finance, 52(3):975-1005.

Andersen, T. G. and Bollerslev, T. (1997b). Intraday periodicity and volatility persistence in financial markets. Journal of Empirical Finance, 4(2-3):115-158.

Andersen, T. G. and Bollerslev, T. (1998a). Deutsche mark-dollar volatility: Intraday activity patterns, macroeconomic announcements, and longer run dependencies. The Journal of Finance, 53(1):219-265.

Andersen, T. G. and Bollerslev, T. (1998b). Towards a unified framework for high and low frequency return volatility modeling. Statistica Neerlandica, 52(3):273-302.

Andersen, T. G., Bollerslev, T., and Cai, J. (2000). Intraday and interday volatility in the japanese stock market. Journal of International Financial Markets, Institutions and Money, 10(2):107-130.

Brock, W. A. and Kleidon, A. W. (1992). Periodic market closure and trading volume: A model of intraday bids and asks. Journal of Economic Dynamics and Control, 16(34):451-489.

Chan, K. C., Christie, W. G., and Schultz, P. H. (1995). Market structure and the intraday pattern of bid-ask spreads for nasdaq securities. The Journal of Business, 68(1):35-60.

Chow, Y.-F., Yung, H. H. M., and Zhang, H. (2003). Expiration day effects: The case of hong kong. Journal of Futures Markets, 23(1):67-86.

Engle, R. F. and Sokalska, M. E. (2012). Forecasting intraday volatility in the US equity market. multiplicative component GARCH. Journal of Financial Econometrics, 10(1):5483. 
Evans, K. P. and Speight, A. E. (2010). Intraday periodicity, calendar and announcement effects in euro exchange rate volatility. Research in International Business and Finance, 24(1):82-101.

Foster, F. D. and Viswanathan, S. (1990). A theory of the interday variations in volume, variance, and trading costs in securities markets. The Review of Financial Studies, $3(4): 593-624$.

Gallant, A. R. (1981). On the bias in flexible functional forms and an essentially unbiased form: The fourier flexible form. Journal of Econometrics, 15(2):211-245.

Harris, L. (1986). A transaction data study of weekly and intradaily patterns in stock returns. Journal of Financial Economics, 16(1):99-117.

ISMR (2010). Indian Securities Market - A Review 2010, volume XIII. National Stock Exchange of India Limited.

ISMR (2011). Indian Securities Market - A Review 2011, volume XIV. National Stock Exchange of India Limited.

Kiymaz, H. and Berument, H. (2003). The day of the week effect on stock market volatility and volume: International evidence. Review of Financial Economics, 12(4):363 - 380.

McInish, T. H. and Wood, R. A. (1990). An analysis of transactions data for the toronto stock exchange: Return patterns and end-of-the-day effect. Journal of Banking and Finance, 14(2 3):441-458.

Muller, U. A., Dacorogna, M. M., Olsen, R. B., Pictet, O. V., Schwarz, M., and Morgenegg, C. (1990). Statistical study of foreign exchange rates, empirical evidence of a price change scaling law, and intraday analysis. Journal of Banking and Finance, 14(6):1189-1208.

NSE (2008). NSE Fact Book - 2008. National Stock Exchange of India Limited.

Stoll, H. R. and Whaley, R. E. (1986). Expiration day effects of index options and futures.

Stoll, H. R. and Whaley, R. E. (1987). Program trading and expiration-day effects. Financial Analysts Journal, 43(2):16-28.

Stoll, H. R. and Whaley, R. E. (1991). Expiration-day effects: What has changed? Financial Analysts Journal, 47(1):58-72.

Taylor, N. (2004a). Modeling discontinuous periodic conditional volatility: Evidence from the commodity futures market. Journal of Futures Markets, 24(9):805-834.

Taylor, N. (2004b). Trading intensity, volatility, and arbitrage activity. Journal of Banking \& Finance, 28(5):1137-1162.

Vipul (2005). Futures and options expiration-day effects: The indian evidence. Journal of Futures Markets, 25(11):1045-1065.

Wood, R. A., McInish, T. H., and Ord, J. K. (1985). An investigation of transactions data for NYSE stocks. The Journal of Finance, 40(3):723-739. 
Figure 1: Intraday Patterns of Trade Size, Volume, No. of Trades and Volatility (Absolute Returns)

The figure shows intraday pattern of trade size, volume, no of transactions and volatility (absolute returns), estimated using NSE trade data for 500 stocks over a period of 108 months (2001 to 2009). Each trading day of 335 minutes is divided into 67 five-minute intervals. The trade size, number of transactions and volume are first standardized (across intervals within a stock-day with mean 0 and standard deviation 1) over the 67 intervals of a particular stock-day and then averaged.

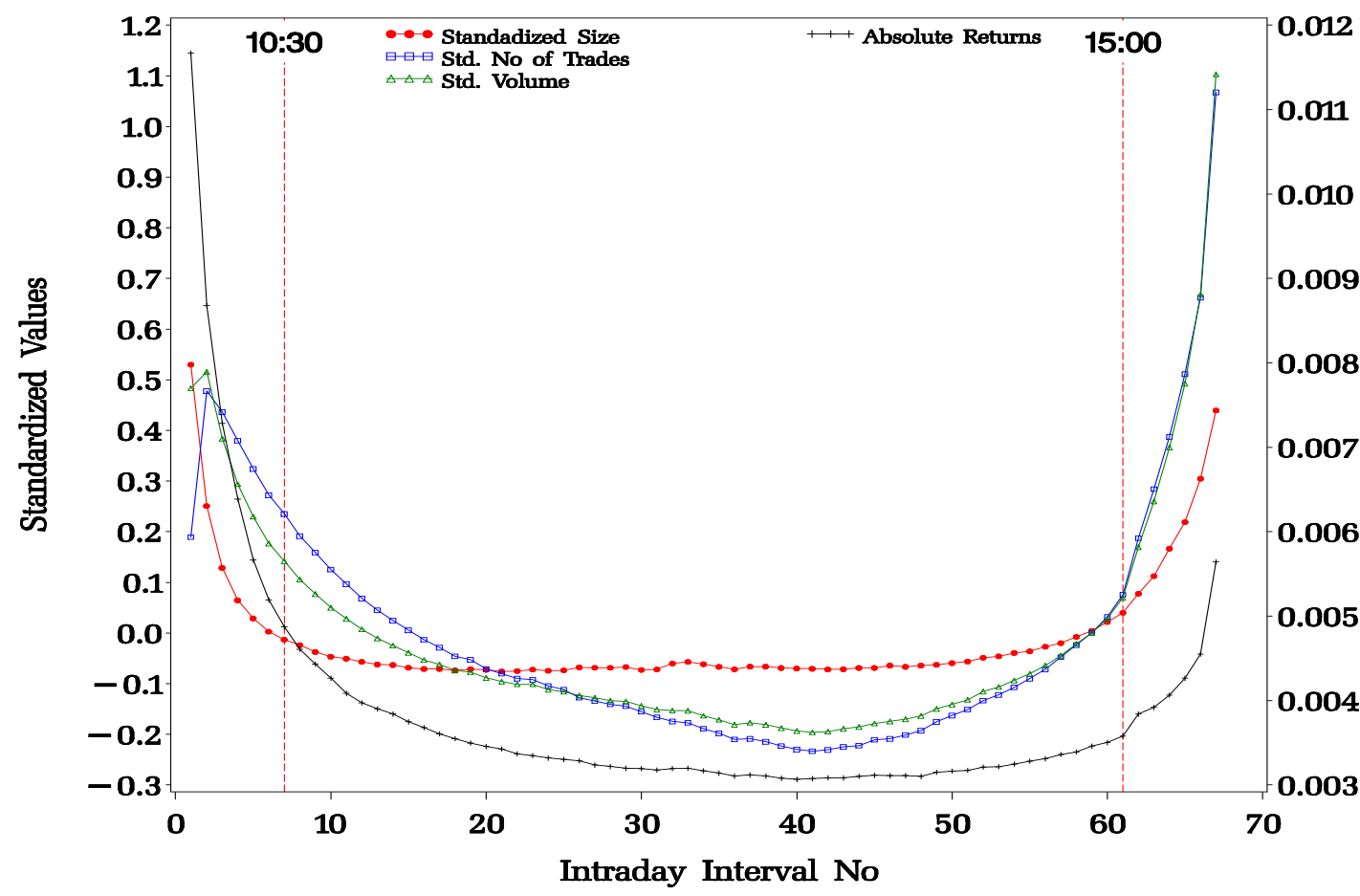


Figure 2: Correlogram for Actual and Filtered Absolute Excess Returns

The six correlograms shows the autocorrelation up to 603 lags (equivalent to 9 trading days) for the series of average actual and filtered volatility (absolute five-minute demeaned returns) i.e. $\left|R_{m, t, n}-\bar{R}_{m}\right|$ and $\left|R_{m, t, n}-\bar{R}_{m}\right| / S_{t, n}$ where $R_{m, t, n}$ is the $\log$ return of stock $m$ at the $n^{t h}$ five-minute interval of day $t$ and $\bar{R}_{m}$ is the mean five-minute returns of each company over the entire period. The series consists of 149,410 (2230 trading days * 67 intervals) intraday average volatilities, of the entire sample and across each M-cap quintiles. $S_{t, n}$, denoted as $f(\theta ; t, n)$, where $\theta$ is the vector of right hand side coefficients, is a function of the following parametric representation: $\mu+\sum_{k=1}^{K} \lambda_{k} I_{k(m, t, n)}+\sum_{p=1}^{P}\left[\delta_{c, p} \cdot \cos \left(\frac{2 \pi p n}{N}\right)+\delta_{s, p} \cdot \sin \left(\frac{2 \pi p n}{N}\right)\right]$. The sinusoid terms capture the smooth variations in intraday pattern. The $I_{k(m, t, n)}$ terms represent $K$ dummy variables, each representing specific events (described in subsection 3.3) occurring in the $(t, n)$ interval for stock $m$. Parameter coefficients $\lambda_{k}$ quantify the impact of specific events, whereas the overall shape of intraday pattern is captured by $\delta$ coefficients. The coefficient are estimated using an OLS regression of the following form: $2 \log \left|R_{m, t, n}-\bar{R}_{m}\right|-\log \hat{\sigma}_{m, t}^{2}+\log N=c+f(\theta ; t, n)+\hat{u}_{m, t, n}$, where $\hat{u}_{m, t, n}$ is a stationary error term and $N$ represents number of intervals in a trading day (67 in our case).
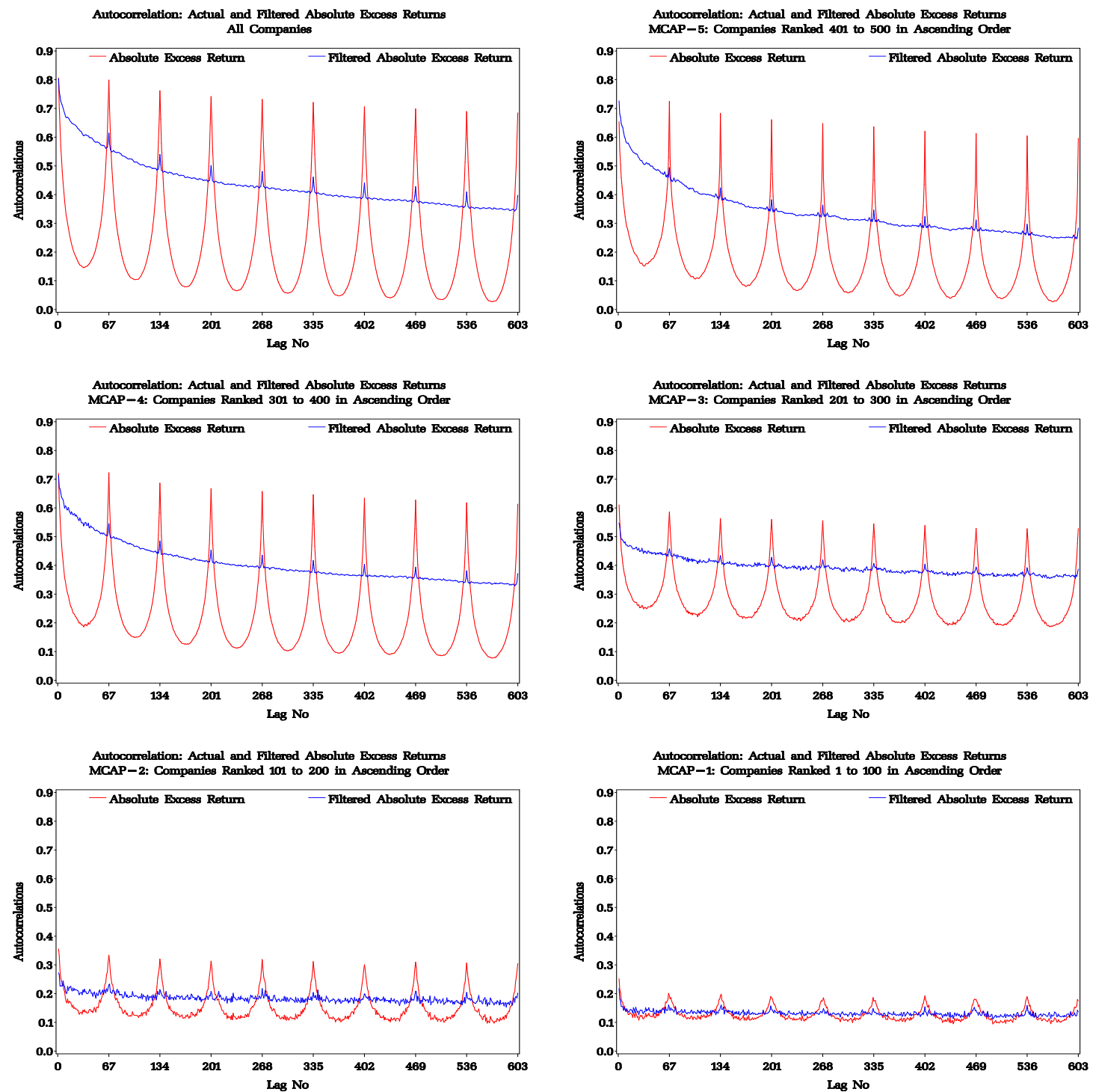
Figure 3: Actual and FFF Fit for Intraday Absolute (Demeaned) Log Returns

The six plots shows average of the actual absolute demeaned five-minute returns i.e. $\left|R_{m, t, n}-\bar{R}_{m}\right|$ and corresponding estimates obtained from the FFF regression model for the entire sample of 500 stocks and for each of the five quintiles over a period of 2230 trading days (9 years). The FFF model is of the following form: $2 \log \left|R_{m, t, n}-\bar{R}_{m}\right|-\log \hat{\sigma}_{m, t}^{2}+\log N=c+f(\theta ; t, n)+\hat{u}_{m, t, n}$, where $R_{m, t, n}$ is the $\log$ return of stock $m$ at the $n^{t h}$ five-minute interval of day $t$ and $\bar{R}_{m}$ is the mean five-minute returns of each company over the entire period, $\hat{u}_{m, t, n}$ is a stationary error term and $N$ represents number of intervals in a trading day (67 in our case). $f(\theta ; t, n)$, where $\theta$ is the vector of right hand side coefficients, is a function of the following parametric representation: $\mu+\sum_{k=1}^{K} \lambda_{k} I_{k(m, t, n)}+\sum_{p=1}^{P}\left[\delta_{c, p} \cdot \cos \left(\frac{2 \pi p n}{N}\right)+\delta_{s, p} \cdot \sin \left(\frac{2 \pi p n}{N}\right)\right]$. The sinusoid terms capture the smooth variations in intraday pattern. The $I_{k(m, t, n)}$ terms represent $K$ dummy variables, each representing specific events (described in subsection 3.3) occurring in the $(t, n)$ interval for stock $m$. Parameter coefficients $\lambda_{k}$ quantify the impact of specific events, whereas the overall shape of intraday pattern is captured by $\delta$ coefficients.
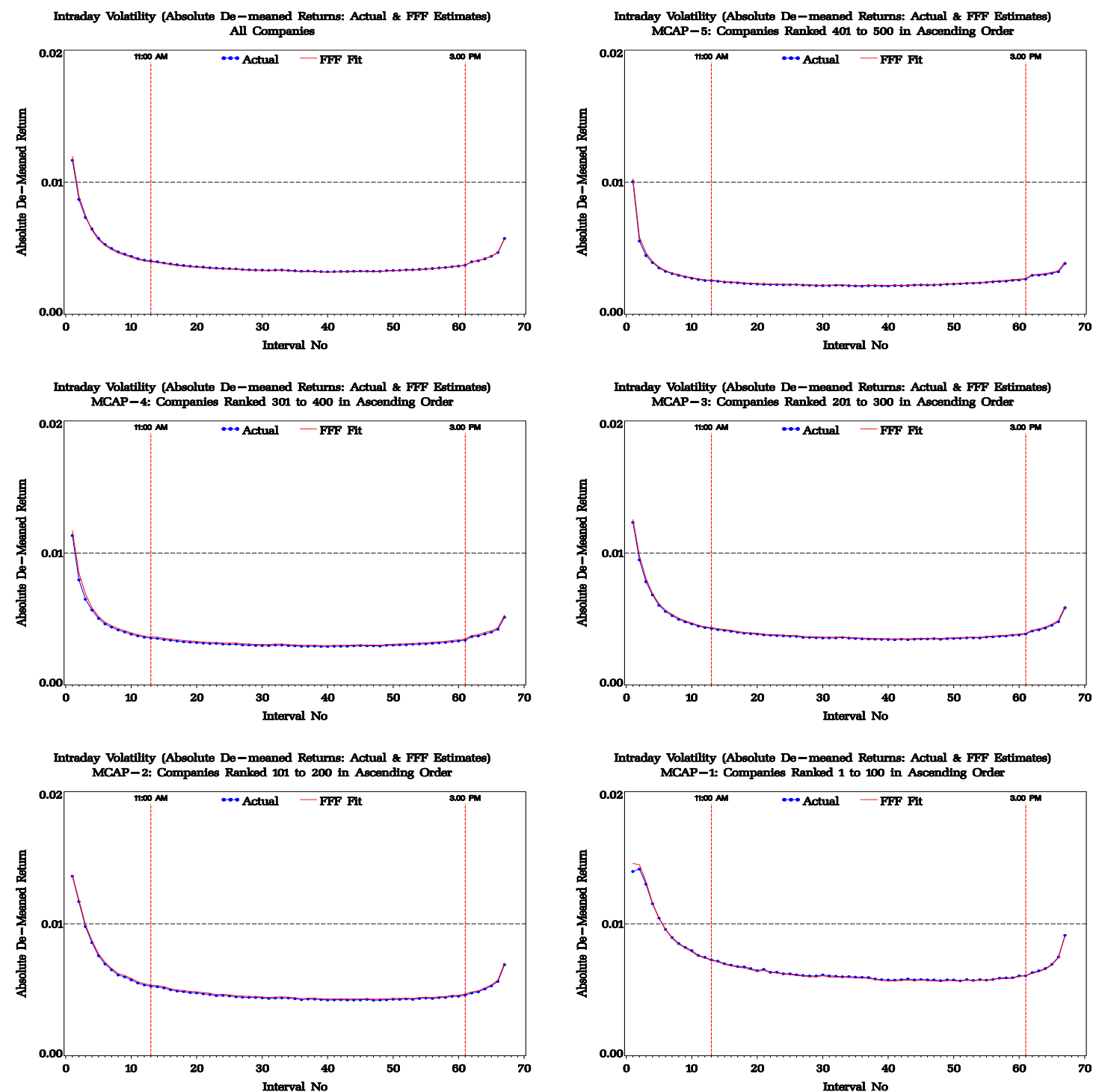


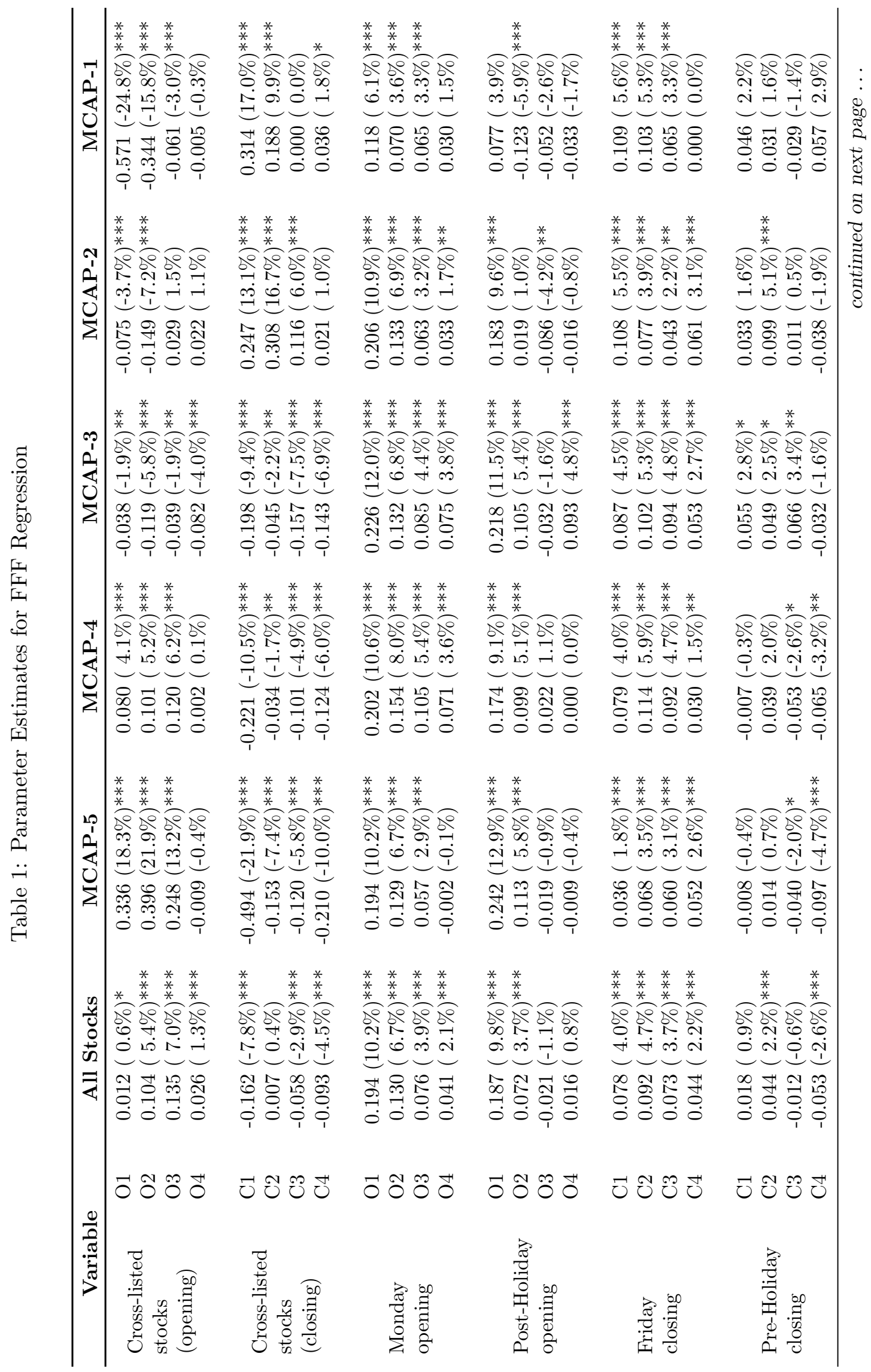




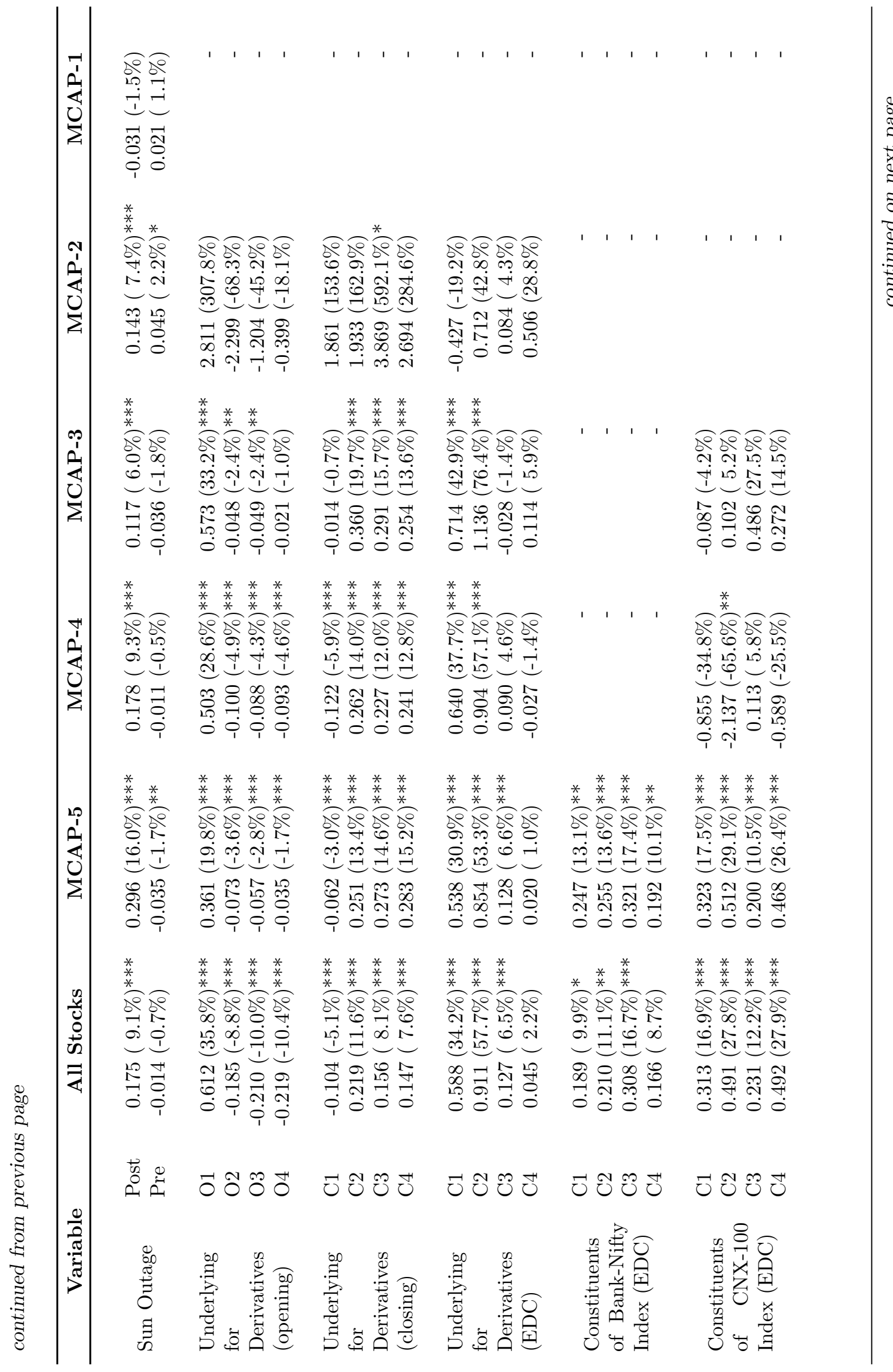




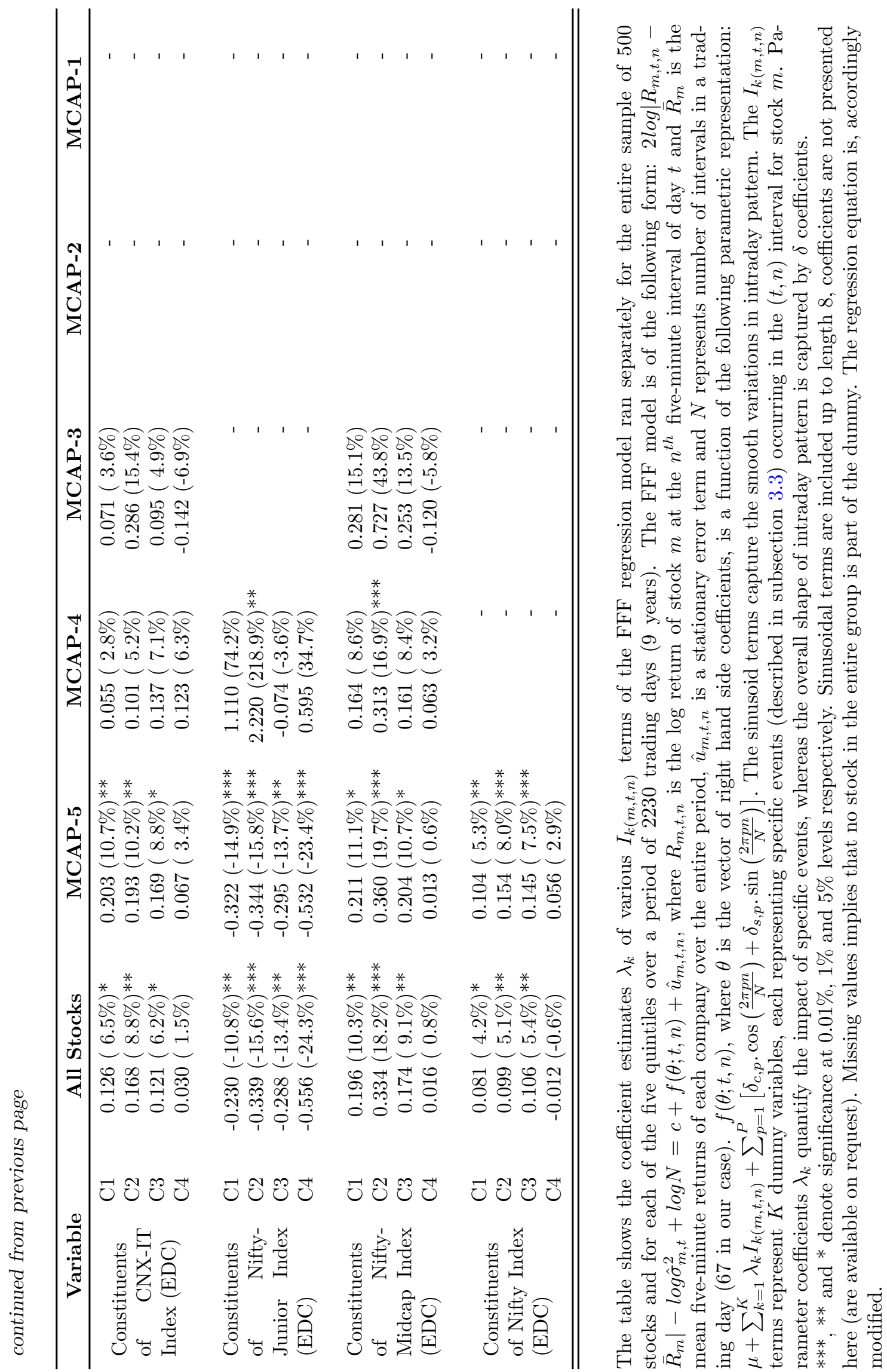

